Original Research Article

\title{
Ultrasonic investigation of molecular interactions in aqueous alkaline solution of mono azo dye metanil yellow and hexacyanoferrate(III) ions
}

Goel, Anjali and Chaudhary, Manu

Depatment of Chemistry, KGC, Gurukul Kangri University, Haridwar, Uttrakhand 249407, India

Corresponding Author: anjali10goel@gmail.com

\section{A R T I C L E I N F O}

Received: 15 May 2018 | Accepted: 25 July 2018 | Published Online: 31 December 2018

DOI: $10.31786 / 09756272.18 .9 .2 .201$

EOI: 10.11208/essence.18.9.2.201

Article is an Open Access Publication.

This work is licensed under Attribution-Non Commercial 4.0 International

(https://creativecommons.org/licenses/by/4.0/)

CThe Authors (2018). Publishing Rights @ MANU_ICMANU \& ESSENCE_IJERC.

\section{A B S T R A C T}

In order to investigate the nature of molecular interaction in the reaction mixture of metanil yellow with hexacyanoferrate (III) ions in basic medium, ultrasonic velocity was measured over wide ranges of concentrations. The different acoustic parameters such as adiabatic compressibility ( $\beta \mathrm{ad})$, intermolecular free length $\left(\mathrm{L}_{\mathrm{f}}\right)$, acoustical impedance $(Z)$, relaxation time $(\tau)$ and Gibb's free energy $(\Delta \mathrm{G})$ were calculated at $7 \mathrm{MHz}$ utilizing ultrasonic interferometer at temperature $313 \mathrm{~K}$. The experimental information for ultrasonic velocity $(u)$, viscosity $(\eta)$ and density $(\rho)$ of the solutions for various concentrations have been utilized to ascertain these parameters. The variation of these parameters with concentration of solute indicates the nature of interaction present in the binary mixture. The results are depicted in terms of molecular interaction between the components of the solutions

\section{K E Y W O R D S}

Ultrasonic velocity | Acoustic parameters | Metanil yellow | Hexacyanoferrate (III) ions.

\section{I T A T I O N}

Goel, Anjali and Chaudhary, Manu (2018): Ultrasonic investigation of molecular interactions in aqueous alkaline solution of mono azo dye metanil yellow and hexacyanoferrate (III) ions. ESSENCE Int. J. Env. Rehab. Conserv. IX (1): $1-6$. 


\section{Introduction}

In recent year, the investigations of acoustical properties of fluid arrangements have been observed to be helpful in understanding the molecular interactions in solution. In the basic science ultrasonic waves have obtained the status of an imperative test for the investigation of structure and properties of matter. Since ultrasonic speed is fundamentally identified with the coupling powers between the constituents of the medium, so it is of great degree receptive to the structure and associations present in the liquid system (Arul et al., 2001).

Ultrasonic examinations are broadly utilized to evaluate the thermodynamic properties and to anticipate the intermolecular communications in pure liquid (Nithiyanantham et al., 2005), liquid mixture (Nithya et al., 2009 and Santhi et al., 2010) and ionic interactions in electrolytic solution (Nath 2012 and Santosh et al., 2010). The estimation of ultrasonic speed of sound in liquids empowers determination of some valuable acoustic and thermodynamic parameters that are observed to be extremely touchy to molecular interaction (Mehra et al., 2000). Derived parameters from ultrasonic speed estimation gives subjective data with respect to the nature and quality of interaction in liquid mixtures (Santosh et al., 2010).

There has been impressive advancement in the assurance of thermodynamic, acoustic and transport properties of liquid frameworks from density and viscosity estimation in recent years. Viscosity, density estimations and the properties got from these are good devices to identify solute - solute and solute - solvent interaction. The accurate estimation of density, viscosity, ultrasonic speed, adiabatic compressibility, intermolecular free length and related parameters give critical data in regards to the situation in an answer. Ultrasonic speeds of the liquid mixtures comprising of polar and non-polar (Mehra et al.,
2007) segments are of extensive centrality in understanding intermolecular interaction between component molecule and discover applications in various industries and technological procedures (Pal et al., 2004 and Halder et al., 2017 ). Sharma et al., (2013 \& 2014) discussed the ultrasonic investigation based on the moleculer interaction of $\mathrm{ZnSO} 4$ in aqueous solution of glucose at various concentration and aqueous solution of 1 , 4 dioxane.

In perspective of the above view point we have completed a methodical exploratory examination of the ultrasonic velocity, density and viscosity measurement of reaction mixture of dye, metanil yellow and hexacyanoferrate (abbreviated as HCF) (III) ions in basic medium. To think about the solute solvent interaction the concentration of reaction mixture was changed by changing the concentration of metanil yellow and hexacyanoferrate(III) ions.

\section{Experimental Section}

All the chemicals and reagents used were of analytical grade. Azo dye metanil yellow was purchased from Loba Chemie (Loba Chemie Pvt. Ltd, Mumbai, India) and potassium hexacyanoferrate(III) from Merck. Deionized water was used throughout the experiments. Single crystal interferometer with frequency $7 \mathrm{MHz}$ was used for the measurement of ultrasonic velocities of the solutions [15]. The reaction mixture was analyzed for the following parameters.

(1) Density Measurement: The density or more precisely, the volumetric mass per unit volume of various solutions of dye and HCF (III) ions were estimated by particular gravity bottle utilizing the accompanying equation.

$$
\rho 2=(w 2 / w 1) \rho 1
$$

where, $w 1$ and $w 2$ are the weight of distilled water and experimental liquid respectively and $\rho 1$ and $\rho 2$ are the density of water and experimental liquid respectively. 
(2) Viscosity Measurement: Viscosity is the measure of a substance's resistance to motion under an applied force. The viscosity of the aqueous solutions is measured using an Ostwald's viscometer calibrated with doubly distilled water. The flow time was measured by a digital stop watch. The viscosity can be calculated using the formula

$\eta 2=\eta 1(\mathrm{t} 2 / \mathrm{t} 1)(\rho 2 / \rho 1)$

where,

$\eta 1$ and $\eta 2$ are the viscosity of water and experimental liquid respectively.

$\mathrm{t} 1, \mathrm{t} 2$ are the time of flow of water and the experimental liquid respectively.

$\rho 1, \rho 2$ are the density of water and experimental liquid respectively.

\section{(3) Ultrasonic Velocity Measurements:} Ultrasonic velocity for different solutions was measured using single crystal interferometer with frequency $7 \mathrm{MHz}$. The principle used in the measurement of velocity ' $u$ ' is based on the accurate determination of the wavelength ' $\lambda$ ' in the medium. Ultrasonic waves of known frequency ' $f$ ' are produced by a quartz plate fixed at the bottom of the cell. The waves are reflected by a movable metallic plate kept parallel to quartz plate. Ultrasonic velocity can be calculated by using the following relation of wavelength and frequency-

$$
\mathrm{u}=\lambda \times \mathrm{f}
$$

After calculating the value of density, ultrasonic velocity and viscosity parameters other parameters and other parameters like adiabatic compressibility ( $\beta \mathrm{ad})$, intermolecular free length $\left(L_{f}\right)$, relaxation time $(\tau)$ and Gibb's free energy $(\Delta G)$ have been calculated as follows.

a) Adiabatic compressibility ( $\beta \mathrm{ad})$ -

Adiabatic compressibility was calculated by using Newton Laplace equation.

$$
\beta \mathrm{ad}=1 / \mathrm{u} 2 \rho
$$

Where, $u=$ velocity $\& \rho=$ density of given solution

b) Intermolecular free length (Lf)-Intermolecular free length was determined by Jacobson formula.

$$
\mathrm{Lf}=\mathrm{KT} \beta \mathrm{ad} 1 / 2
$$

Where $\mathrm{K} \mathrm{T}$ is temperature dependent constant

$$
=(93.875+0.375 \mathrm{~T}) \times 10-8
$$

c) Acoustic Impedance (Z) - Z can be calculated by following equation.

$$
\mathrm{Z}=\mathrm{u} \times \rho
$$

d) The relaxation time $(\tau)$ - The relaxation time was calculated by given relation.

$$
\tau=(4 / 3) \beta \mathrm{ad} \eta
$$

e) Gibb's free energy $(\Delta G)$-Gibbs free energy can be determined from acoustic relexation time as follows-

$$
\Delta \mathrm{G}=\mathrm{RT} \ln (\mathrm{kT} \tau / \mathrm{h})
$$

Where, $\mathrm{k}$ is Boltzmann constant, $\mathrm{T}$ is the absolute temperature and $\mathrm{h}$ is planck's constant.

\section{Results and discussion}

A perusal of the data presented in the Table 1 and 2 show that there is no regular increase or decrease in the value of $\mathrm{u}, \eta, \beta \mathrm{ad}, \mathrm{Lf}, \mathrm{Z}, \tau$ and $\Delta \mathrm{G}$ but the comparison of the data in the range of metanil yellow concentration $1 \times 10-5$ to $8 \times 10-5 \mathrm{~mol} / \mathrm{dm} 3$ reveals an overall increase in $u, \rho, Z$ and $\eta$ and an overall decrease in the value of $\beta \mathrm{ad}, \mathrm{Lf}, \tau$ and $\Delta \mathrm{G}$. The increase of density with increase in concentration of metanil yellow suggesting a strong interaction between solute and solvent. Fig.1 shows the variation of ultrasonic velocity with solute concentration of metanil yellow at constant temperature and $\mathrm{pH}$ of reaction mixture. The ultrasonic velocity initially decreases and exhibits a dip at metanil yellow concentration $4 \times 10-5 \mathrm{~mol} / \mathrm{dm} 3$ after that it increases and goes to maxima at concentration $6 \times 10-5 \mathrm{~mol} / \mathrm{dm} 3$. The adiabatic compressibility shows exactly an opposite behavior as expected (Fig -2). 
Fig. 1: Plot of ultrasonic velocity versus different concentration of MY at $313 \mathrm{~K}$

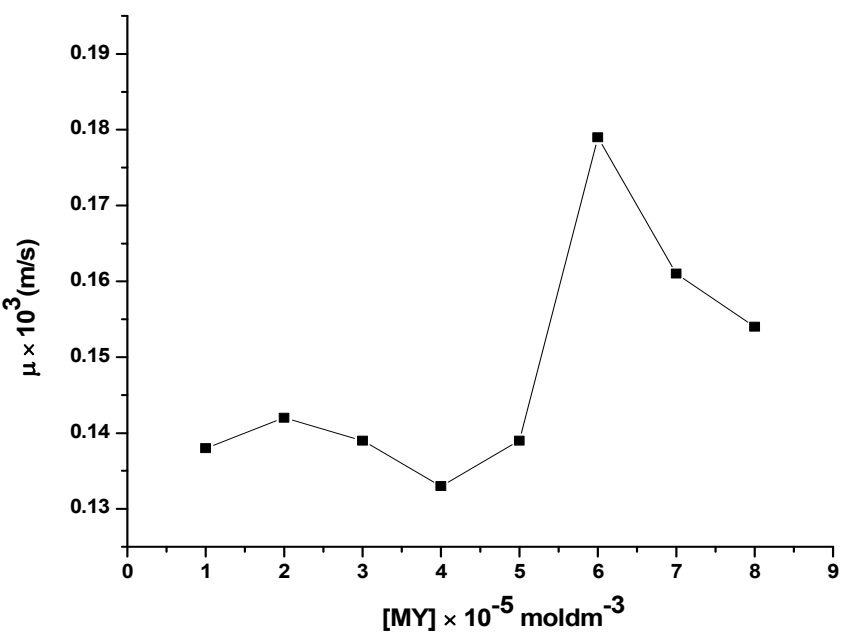

Fig. 2: Plot of adiabatic compressibility versus different concentration of MY at $313 \mathrm{~K}$.

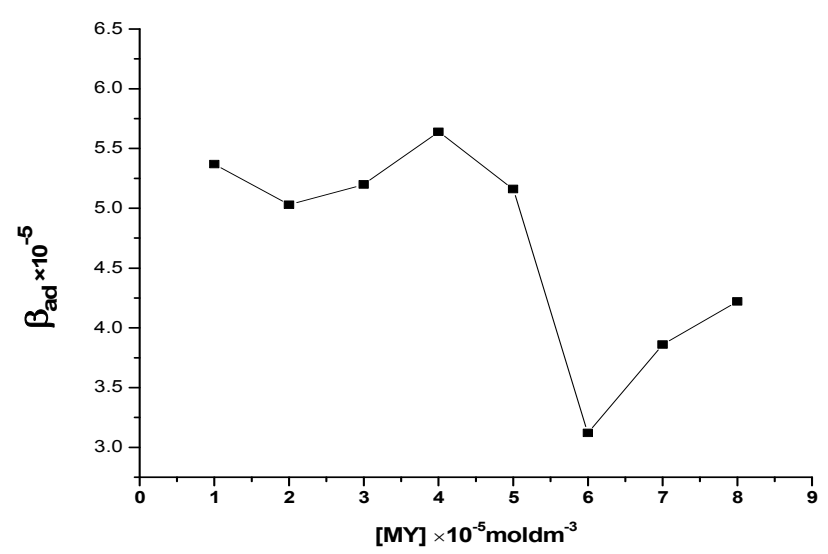

Fig. 3: Plot of viscosity versus different concentration of MY at $313 \mathrm{~K}$

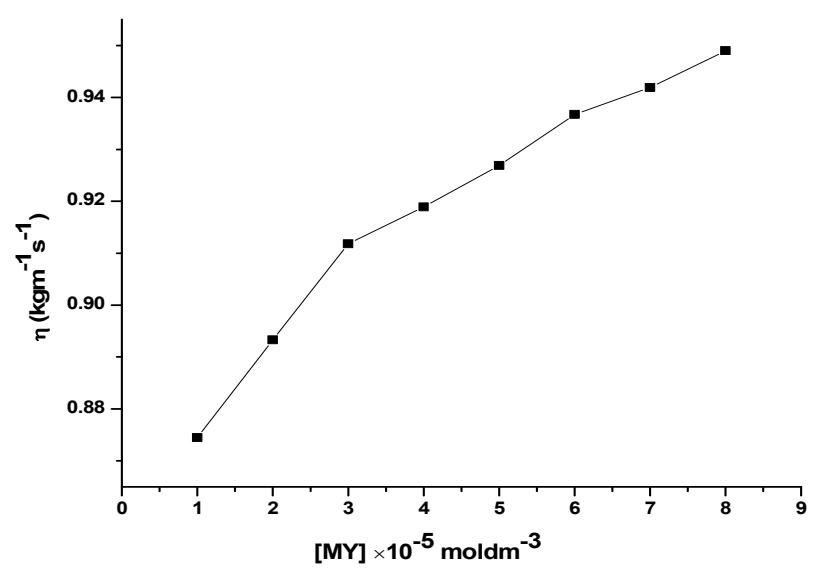

Fig. 4: Plot of density versus different concentration of MY at

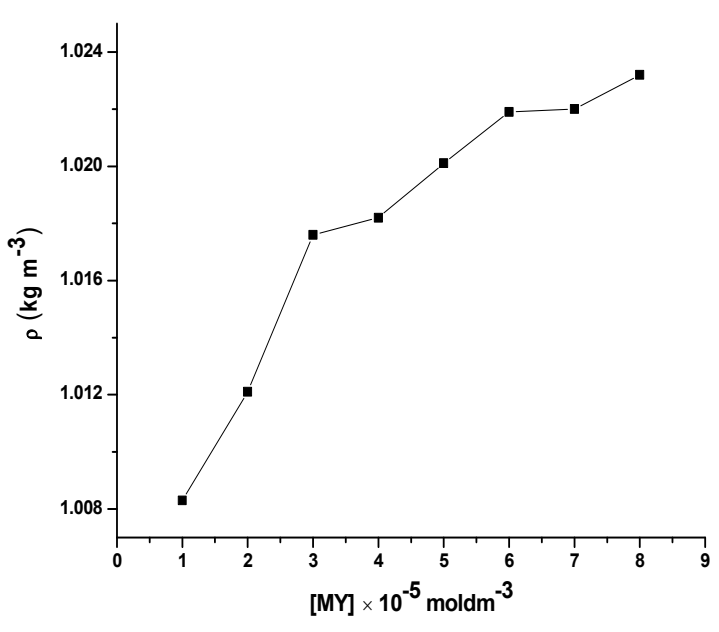

Fig. 5: Plot of intermolecular free length versus different concentration of MY at $313 \mathrm{~K}$.

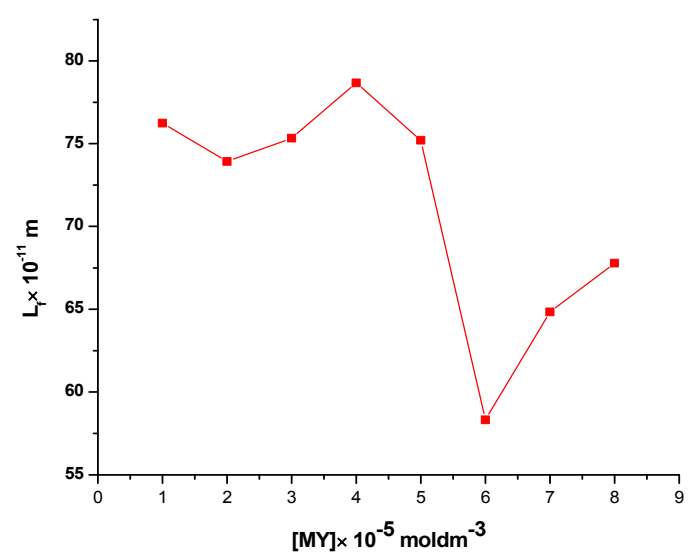

Fig. 6: Plot of Gibbs free energy versus different concentration of MY at $313 \mathrm{~K}$.

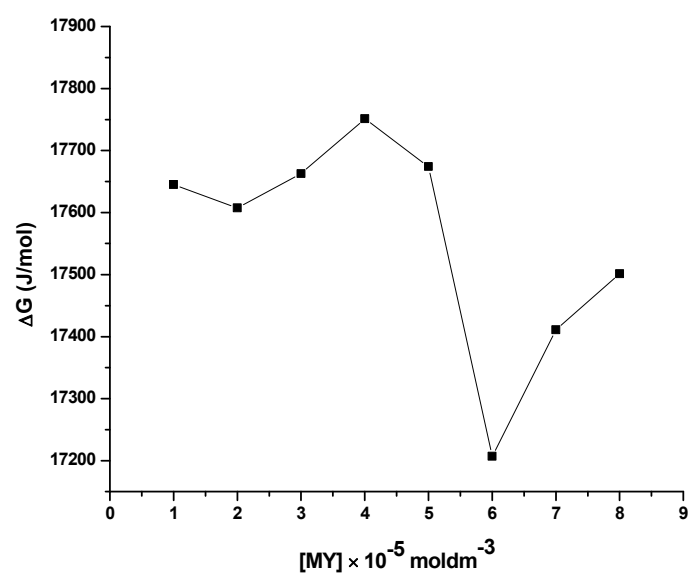


This dip and peak of ultrasonic velocity and adiabatic compressibility at higher or lower metanil yellow concentration in aqueous phase may be due to weak or strong intermolecular forces between solute and solvent. This indicates that at lower concentration, molecular dissociation takes place and weak solute solvent interaction occurs but intermediate concentration of metanil yellow, molecular association and strong solute solvent interaction results. The greater association may result due to hydrogen bonding between solute and solvent molecules. The effect of HCF(III) on the ultrasonic velocity has also been studied by varying it in the range of $1 \times 10-6$ to $8 \times 10-6 \mathrm{~mol} / \mathrm{dm} 3$.The results presented in the form of Table - 3 shows that there is no measurable increase or decrease in the ultrasonic velocity with $[\mathrm{HCF}(\mathrm{III})]$ as ultrasonic velocity changes from 0.140 to 0.141 only. It reveals weak solute interaction of HCF (III) as compared to metanil yellow. is supported by the mechanism of reaction that at lower dye concentration the dissociation of metanil yellow into anion dominates but at higher dye concentration decomposition of complex into products dominates. According to mechanism in alkaline medium dye molecule exists as anion which form a complex compound with hexacyanoferrate (III) ions. Decomposition of complex results in to product. According to the given mechanism, it is assume that dye (MY) molecule exist as an anion in the alkaline medium which forms complex with HCF(III) ions. This complex dissociate through a slow step into product and short lived Fe (CN) 64 .

$$
\begin{gathered}
\mathrm{MY}^{-}+\mathrm{Fe}\left(\mathrm{CN}_{6}{ }^{3-} \underset{\mathrm{K}}{\stackrel{\mathrm{K}}{\rightleftharpoons}} \operatorname{Complex}(\mathrm{C})\right. \\
\operatorname{Complex}(\mathrm{C}) \underset{\text { slow }}{\stackrel{\mathrm{k}_{1}}{\longrightarrow}} \mathrm{IP}
\end{gathered}
$$

$\mathrm{IP}+\mathrm{Fe}(\mathrm{CN})_{6}{ }^{3-} \underset{\text { fast }}{\stackrel{[\mathrm{O}]}{\longrightarrow}} \mathrm{Fe}\left(\mathrm{CN}_{6}{ }^{4}+\right.$ Product
The intermediate product is further oxidized to final product mainly carboxylic acids and alcohols.

\section{Conclusion}

The eventual outcomes of the present examination suggest that the ultrasonic speed and other determined acoustic parameters depend on the concentration of solution due to the presence of molecular communication. A methodical report at variable concentration of metanil yellow with hexacyanoferrate (III) ions in water demonstrate that solute solvent interaction due to hydrogen bonding increases with the increase of concentration of metanil yellow. It is maximum at about intermediate concentration of $6 \times 10-5$ $\mathrm{mol} / \mathrm{dm} 3$ and minimum at $4 \times 10-5 \mathrm{~mol} / \mathrm{dm} 3$. Thus experimental parameters give the important data in regarding ion dissolvable interaction in aqueous solution.

\section{Acknowledgement}

The authors are thankful to Department of Chemistry, KGC, Gurukul kangri University Haridwar for experimental support.

Conflict of Interest: No competing financial interest exist.

\section{References}

Arul, G. and Palaniappan, L. (2001): Molecular interaction studies in the ternary mixture of cyclohexane+ toluene+ 2-propanol. Indian J. Pure Appl. Phys. 39, 561-564.

Fort, R. J. and Moore, W. R. (1966): Viscosities of binary liquid mixtures. Transactions of the Faraday Society, 62, 1112-1119.

Gupta, V.; Sharma, A. K. and Sharma, M. (2013): Ultrasonic investigation of molecular interaction in aqueous solution of 1, 4dioxane. Journal of Biosphere, 2(1): 2831.

Gupta, V.; Sharma, A . and Sharma, M. (2014): Ultrasonic investigation of molecular interaction in aqueous glycerol and 
aqueous ethylene glycol solution. Journal

of Chemical and Pharmaceutical Research, 6(1): 714-720.

Halder, S.; Thakur, S. and Agrawal, P. S. (2017):

Ultrasonic Study of Molecular Interactions in ternary liquid mixture of 1, 4-Bis (diphenylaminobenzene) with 3Nitrobenzoic acid And Picric acid in ethanol at $298 \mathrm{~K}$. Advance and Innovative Research, 5.

Mehra, R. and Pancholi, M. (2007): Study of molecular interactions in binary mixtures of benzene-butanol and toluene-butanol systems from acoustic and thermodynamic parameters. Indian Journal of Pure and Applied Physics. $45,580-590$.

Mehra, R. and Sajnani, H. (2000): Acoustical studies in ternary electrolytic mixtures at 25, 30, 35, 40 and 45 C. Indian J. Pure Appl. Phys., 38(11): 762-765.

Santhi, N.; Sabarathinam, P. L.; Gopi, C.; Emayavaramban, M. and Manivannan, C. (2010): Molecular interaction studies in binary liquid mixtures from ultrasonic data. Journal of Chemistry, 7(2): 648-654.
Nath, G. (2012): Ultrasonic Study of Binary Mixture of Acetone with Bromobenzene and Chlorobenzene at Different Frequencies. Chem. Sci. Trans. 1, 516521.

Nithiyanantham, S. and Palaniappan, L. (2005): Ultrasonic Study of Molecular Interactions in Binary Mixtures at $303 \mathrm{~K}$, Acta Ciencia Indica, 34(1): 533-538.

Nithya, R.; Nithiyanantham, S.; Mullainathan, S. and Rajasekaran, M. (2009): Ultrasonic investigation of molecular interactions in binary mixtures at $303 \mathrm{~K}$. Journal of Chemistry, 6(1): 138-140.

Pal, A. and Kumar, H. (2004): Temperature dependence of the volumetric properties of some alkoxypropanols + n-alkanol mixtures. The Journal of Chemical Thermodynamics, 36(3): 173-182.

Santosh, M. S.; Bhatt, A. S. and Bhat, D. K. (2010): Physico-chemical, acoustic and excess properties of glycylglycine$\mathrm{MnCl} 2$ in aqueous ethanol mixtures at different temperatures. Fluid Phase Equilibria, 291(2): 174-179. 